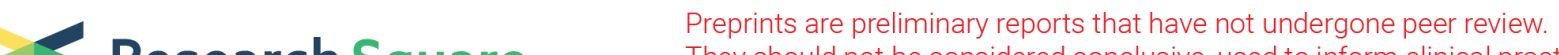 Research Square They should not be considered conclusive, used to inform clinical practice, or referenced by the media as validated information.
}

\section{A Case Report: Angioimmunoblastic T Cell Misdiagnosed as Pulmonary Sarcoidosis}

\section{xuerui Wang}

Five Branches University

\section{Xu jie}

Qingdao Municipal Hospital Group

\section{Chen guang}

qingdao daxue: Qingdao University

bingyan Zhan ( $\nabla$ byz96@sina.com )

Qingdao Municipal Hospital Group https://orcid.org/0000-0002-3107-709X

\section{Case Report}

Keywords: Angioimmunoblastic T-cell lymphoma (AITL), Sarcoidosis

Posted Date: May 21st, 2021

DOI: https://doi.org/10.21203/rs.3.rs-540042/v1

License: (c) (1) This work is licensed under a Creative Commons Attribution 4.0 International License. Read Full License 


\section{Abstract}

Introduction: Angioimmunoblastic T-cell lymphoma is a peripheral T-cell lymphoma subtype characterized by abnormal proliferation of $\mathrm{T}$ lymphocytes with hyperplasia of endothelial veins and follicular dendritic cells. Sarcoidosis is a non-caseating epithelial granulomatous disease of unknown cause, which can invade the whole body organs, especially the lungs and intrathoracic lymph nodes. The clinical manifestations are not specific. There are many similarities between the malignant lymphoma and pulmonary sarcoidosis in the early clinical manifestations, such as chest imaging and clinical manifestations, which are easy to be misdiagnosed.

Methods: A fifty-three-year-old man presented with a two-month history of cough.

This article uses the clinical data of a patient to reveal the main points that should be paid attention to in the diagnosis of lymphoma.

Results: The patient were given right inguinal lymph node biopsy and bone marrow biopsy.

Case Presentation: Here, A fifty-three-year-old man presented with a two-month history of cough. The effect was not good after one week of anti-infective and glucocorticoid treatment. Lymph node biopsy and bone marrow biopsy were performed.

Conclusion: When we find that the therapeutic effect of pulmonary sarcoidosis is not good, we should make pathological diagnosis. We can prevent further deterioration of the disease through the above measures.

\section{Introduction}

Angioimmunoblastic T-cell lymphoma (AITL) is a subtype of peripheral T-cell lymphoma characterized by abnormal proliferation of T lymphocytes with high endothelial vein (HEV) and follicular dendritic cell (FDC) proliferation, accounting for $1-2 \%$ of non-Hodgkin's lymphomas account for $19-20 \%$ of peripheral T-cell lymphomas [1]. Compared with western countries, the incidence rate in my country is higher [2]. AITL is mostly related to abnormal immune function, with complex clinical manifestations, unique pathological characteristics, large heterogeneity, and poor overall prognosis.

In most cases of pulmonary sarcoidosis, bilateral hilar lymph nodes are symmetrically enlarged, or accompanied by mediastinal lymph node enlargement, and the enlarged lymph nodes are confined to the hilar and beside the trachea. It is particularly important for the early diagnosis of the disease. At the same time, the disease is easily confused with pulmonary sarcoidosis. The application of necessary diagnosis and treatment methods is of great significance for the diagnosis of pulmonary lymphoma.

\section{Methods}


A fifty-three-year-old man presented with a two-month history of cough. The patient is accompanied by sputum expectoration without hemoptysis. He had a fever for 1 day and body temperature was up to 37.8 degrees Celsius. He had intermittent taken oral cefpodoxime proxetil and paracetamol for the above symptomsat, but the effect is not good. He denied any tobacco or alcohol use. His family was not notable for heart disease, hypertension or diabetes.

Physical examination: Middle-aged male, He is conscious. A rash was seen in the extremities. Enlarged several lymph nodes were touched in bilateral groin and armpit. Bilateral auscultation could be heard moist rales thick breath sound in both lungs. His heart rate 90 beats / min, rhythm, no pathological murmur was heard in each valve auscultation area. The abdomen is flat and soft. The liver and spleen are not touched under the ribs. There was no edema in both lower limbs.

Laboratory findings showed white blood cell count $14.1 \times 10^{9} / \mathrm{L}$, Tumor markers: Carbohydrate antigen 12 - 5: $59.65 \mathrm{U} / \mathrm{ml}$ (normal range: 0-27 U/ml), Neuron specific enolase18.82ng/ml (normal range: 0-15 $\mathrm{ng} / \mathrm{ml}$ ). Arterial blood gas analysis: P02: $65 \mathrm{mmHg}, \mathrm{PCO} 2: 36 \mathrm{mmHg}, \mathrm{PH}: 7.42$. Liver function, renal function, blood electrolyte, myocardial enzyme and B-type natriuretic peptide were generally normal.

Chest computerized tomographic scanning: symmetrical enlargement of bilateral hilar lymph nodes and pulmonary interstitial changes [Fig. 1]. Upper abdominal color ultrasound: Enlargement of liver and spleen.

The patient was initially diagnosed as pulmonary sarcoidosis. Cefoperazone sodium and sulbactam sodium $3 \mathrm{~g}$ q8h and methylprednisolone $40 \mathrm{mg}$ qd were taken medication to this patient for a week. Cough is not getting better than before. Chest tightness is more serious than before. Laboratory findings showed white blood cell count 10.8×109/L. Arterial blood gas analysis: PO2: $53 \mathrm{mmHg}, \mathrm{PCO}: 42 \mathrm{mmHg}, \mathrm{PH}: 7.40$. The patient were given right inguinal lymph node biopsy [Fig. 2, 3] and bone marrow biopsy [Fig. $4-1$, Fig. 4 - 2].

The normal structure of the submitted lymph node was destroyed, and many small lymphocytes with a single shape were diffusely proliferated and infiltrated, and the cytoplasm was lightly stained and translucent, which were tumor cells. There were also large-sized immunoblasts scattered and distributed. High endothelial blood vessels were seen in the interstitium Irregular hyperplasia with infiltration of eosinophils. Immunohistochemical staining showed tumor cells: CD3 (+), CD4 (+), CD10 (+), PD1 (+), CXCL13 (+), CD21 showed enlarged and destroyed follicular dendritic network. Supports angioimmunoblastic T-cell lymphoma. Final diagnosis: Angioimmunoblastic T-cell lymphoma (AITL).

We had adjusted the treatment plan in time. We made application of E-cyclophosphamide (cyclophosphamide, epirubicin, vincristine, etoposide, prednisone) for 3 cycles. The patient's condition is better than before. [Fig. 5]

Fig2-1, 2-2, 2-3: CD20 (stove shape, positive), CD3 (negative), CD4 (partly, positive), CD8 (partly, positive), CXCL-13 (scattered, positive), PD-1 (scattered, positive), Ki-67 (positive, 30\%), EBER (In situ hybridization, 
negative), p53 (partly, positive), CD10 (partly, positive).

\section{Discussion}

AITL is more common in middle-aged and elderly men, the etiology is unknown, may be related to viral infection, allergic reactions, often have fever, rash, systemic lymph node enlargement, hepatosplenomegaly and other systemic symptoms, most of the patients are accompanied by abnormal immune function. The clinical manifestations of AITL are complex and changeable, often systematic, with large individual differences. Many patients mainly manifest fever, anemia, pruritus and systemic rash, even in dermatology for many years, until lymph nodes are enlarged. AITL is easy to be missed and misdiagnosed. $80 \%$ of the patients are in clinical stage $\nabla \sim$ when the diagnosis is clear. The clinical characteristics of AITL can be summarized as systemic multi-system involvement caused by abnormal immune function, mainly as follows: (1) B symptoms: non-specific symptoms, such as fever, itching, rash, polyarthritis, anemia, body cavity effusion and so on. (2) Systemic lymphoid tissue involvement: systemic lymph node enlargement, hepatosplenomegaly, bone marrow involvement and so on. (3) Abnormal laboratory examination: lymphopenia, thrombocytopenia and accelerated erythrocyte sedimentation rate, eosinophil increase, $\beta$ 2-microglobulin and lactate dehydrogenase increase. (4) Complicated with other tumors or syndromes. It has been reported that AITL was accompanied by Churg's syndrome, primary gastric cancer and chylous ascites. (5) The clinical course of AITL varies greatly: there are reports of spontaneous remission. Some of the pathogenesis is slow and some of the clinical processes are invasive, but most studies show that the prognosis of the disease is very poor.

The lymph nodes involved in AITL had the following morphological characteristics [3, 4]: 1) the structure of lymph nodes was destroyed in varying degrees, some of them had residual marginal sinus or follicles, branched blood vessels proliferated significantly, and some patients had hyaline degeneration of vascular wall with deposition of glycogen staining positive materials. 2) Lymphoma cells vary in size, with rich cytoplasm, light staining, pleomorphic or round nuclei and fine chromatin. 3) Plasma cells, eosinophils, reactive small lymphocytes and histiocytes are scattered. Tumor cells express specific markers: tumor cells express $T$ cell differentiation antigen CD3, Most tumor cells express CD4, not as much as CD7 and CD8, express CD10, CXCL13, Bc-16 and PD-1 at the same time. The histomorphology and immunohistochemistry of lymph nodes in this patient were consistent with the typical AITL characteristics. Positive CD10 is an important phenotype in the diagnosis of AITL and extranodal lesions. The expression of CXCL13 is helpful to describe the morphological spectrum of AITL, which further supports that AITL comes from helper T cells. The results of lymph node imprint in this case are consistent with the pathological results, which is of reference value for the rapid diagnosis and differential diagnosis of malignant lymphoma.

The main points of differentiation from pulmonary sarcoidosis and AITL are: (1) Both of them may have intrathoracic lymph node involvement and superficial lymph node enlargement, but lymphoma clinically has fever, emaciation, anemia, hepatosplenomegaly, and obvious symptoms of mediastinal compression, such as irritant cough and wheezing caused by tracheal pressure, superior vena cava syndrome, 
hoarseness caused by mediastinal nerve compression. The clinical symptoms of sarcoidosis are few and mild. (2) The lymph nodes of patients with lymphoma were gradually enlarged and fused into masses, mainly paratracheal lymph nodes, often involved retrosternal lymph nodes at the same time. Chest X-ray films often show unilateral or bilateral asymmetrical hilar lymph nodes enlarged, hilar lymph nodes tend to fuse with the right cardiac margin [5]. Lateral films show that enlarged mediastinal lymph nodes fill the anterior superior mediastinum area, which is the characteristic of lymphoma. The vast majority of bilateral hilar lymph nodes in sarcoidosis are symmetrically enlarged, or with enlarged mediastinal lymph nodes. The enlarged lymph nodes are limited to the hilum and paratrachea, often separated from the right cardiac margin, and each enlarged lymph node can be identified. After sarcoidosis appeared in the lung, the intrathoracic lymph node enlargement stopped developing, shrinking or dissipating, while malignant lymphoma showed pathological changes in the lung, the enlarged lymph nodes continued to develop and enlarge [6]. (3) Tissue biopsy and Kveim skin test are the main differential methods, and the positive rates of superficial lymph node biopsy and mediastinoscopic lymph node biopsy are higher.

The early clinical manifestation of this patient was not significantly specific to pulmonary sarcoidosis. Although the chest CT showed symmetrical hilar lymph node enlargement and pulmonary interstitial manifestations, it was difficult to differentiate from II stage pulmonary sarcoidosis. However, the hilar lymph nodes in chest CT tended to fuse, and the condition worsened after glucocorticoid treatment. It is suggested that the diagnosis should be confirmed by pathological biopsy of lymph nodes.

HE staining and immunohistochemical staining of lymph nodes have diagnostic value for this patient, and many pathological indexes are consistent with AITL.

In case of superficial lymph node enlargement and fever, lymph node biopsy should be performed early to avoid misdiagnosis or delayed diagnosis of malignant diseases.

AITL is a unique subtype of T-cell lymphoma, and there is no standard treatment at present. At present, CHOP chemotherapy is still used as a first-line treatment, supplemented by local radiotherapy, autologous stem cell transplantation and so on. However, the therapeutic effect of $\mathrm{CHOP}$ regimen is poor, and the recurrence rate is high and the maintenance time is short [7]. Because AITL is often accompanied by abnormal immune function, which leads to higher infection complications, which may be the reason for the poor effect of chemotherapy. The patient's condition improved after 3 cycles of chemotherapy with modified $\mathrm{CHOP}$ regimen.

\section{Declarations}

Ethics approval and consent to participate: The patient agreed to publish the report.

Consent for publication: Consent was obtained by all participants in this study.

\section{Availability of data and materials}


Funding: no

Authors' contributions: bingyan Zhan contributed to the conception of the study; Xuerui Wang performed the experiment; Jie Xu and Chen gaung contributed significantly to analysis and manuscript preparation; Xuerui Wang performed the data analyses and wrote the manuscript;

\section{Acknowledgements: no}

The authors have declared that no competing interests exist.

Ethical conflict: Consent was obtained by all participants in this study.

\section{References}

[1] SWERDLOW S H, CAMPO E, PILERI S A, et al. The 2016revision of the World Health Organization classification of lymphoid neoplasms囚J囚.Blood, 2016, 127(20): 2375-2390.

[2] Swerdlow SH, Harris NL, Jafe ES, et al. WHO classification of tumours of haematopoietic and lymphoid tissues[M]. Lyon: IARC Press, 2017: 408®410.

[3] Jafe ES, Harris NL, Stein H, et al. World Health Organization classification of tumour: pathologyandgenetics of tumors of hematopoietic and lymphoid tissue [M]. Lyon: IARC, 2001: 204『207.

[4] Swerdlow SH, Harris NL, Jafe ES, et al. WHO classification of tumors of hematopoietic and lymphoid tissues[M]. Lyon: IARC, 2008:308ه310.

[5] Swerdlow SH, Campo E,Pileri SA, et al. The 2016 revision of the World Health Organization classification of lymphoid neoplasms[J]. Blood,2016,127(20):2375囚2390. DOI: 10.1182/ blood $₫ 2016 \rrbracket 01 \rrbracket 643569$.

[6] Touré SA, Seck M, Diallo AB, et al. Diagnosis Dilemma of Angioimmunoblastic T-Cell Lymphoma in Tuberculosis Endemic Region. Case Rep Hematol. 2020 Oct 7;2020:8824843. doi:

10.1155/2020/8824843. PMID: 33083069; PMCID: PMC7563086.

[7] Broccoli A, Zinzani PL. Angioimmunoblastic T-Cell Lymphoma. Hematol Oncol Clin North Am. 2017 Apr;31(2):223-238. doi: 10.1016/j.hoc.2016.12.001. PMID: 28340875.

\section{Figures}




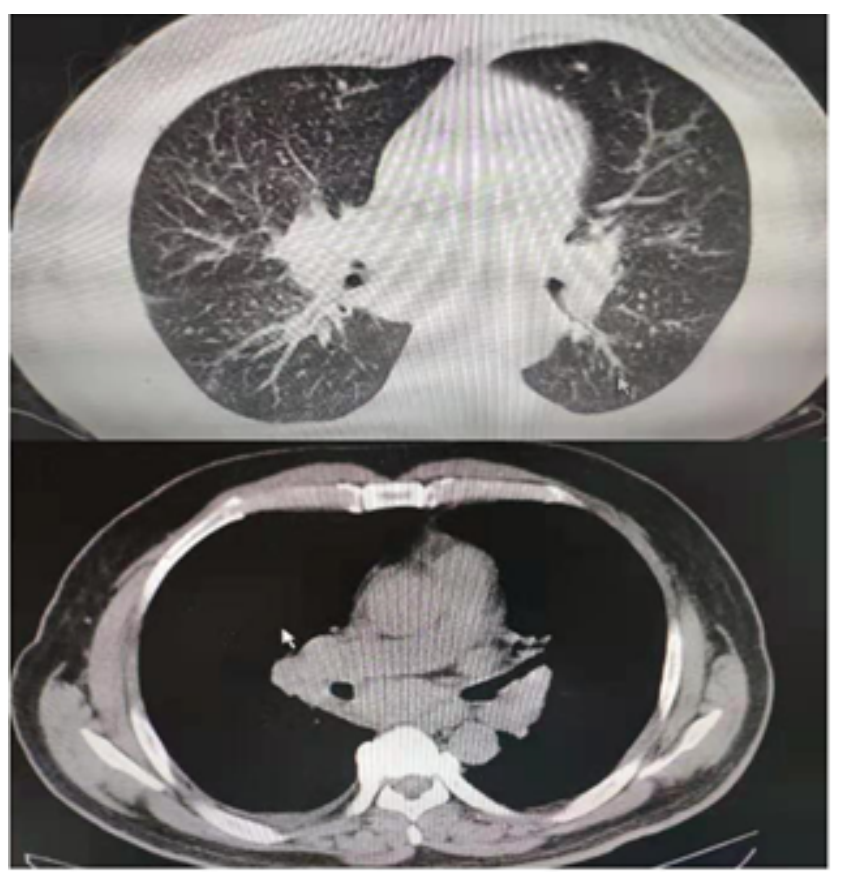

Figure 1

bilateral lymphadenopathy and pulmonary interstitial changes.

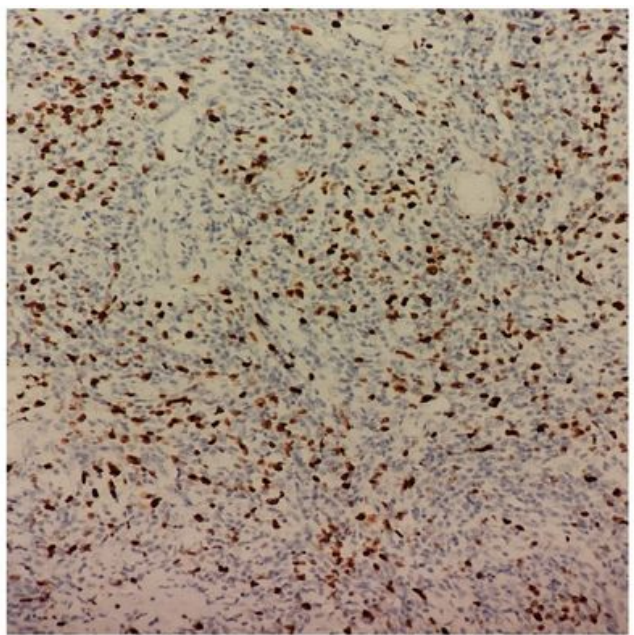

2.1

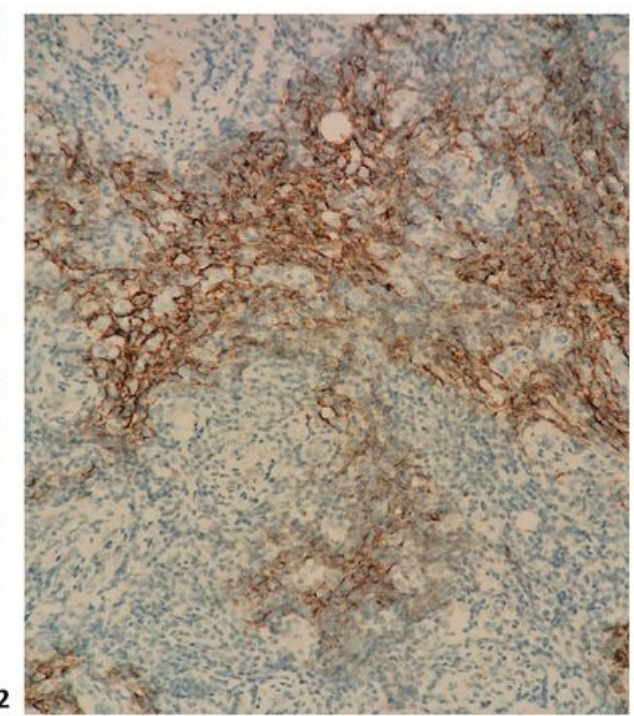

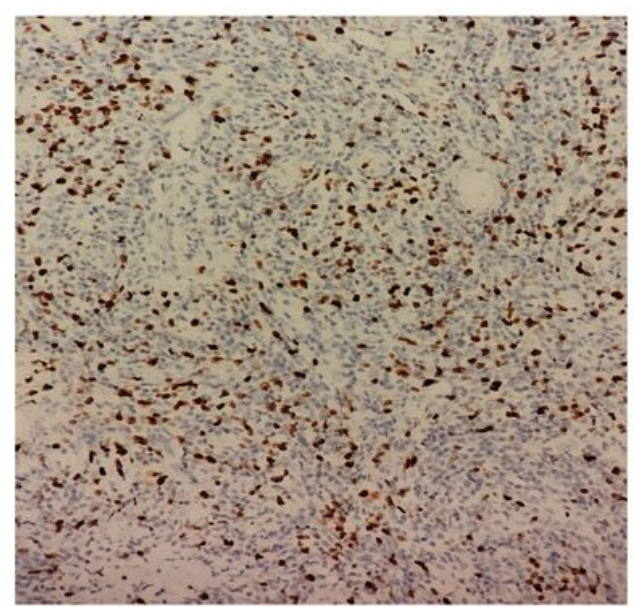

2.3

Figure 2

2-1: Immunohistochemistry (CD4). 2-2: Immunohistochemistry (CD21) 2-3: Immunohistochemistry (Ki67) 


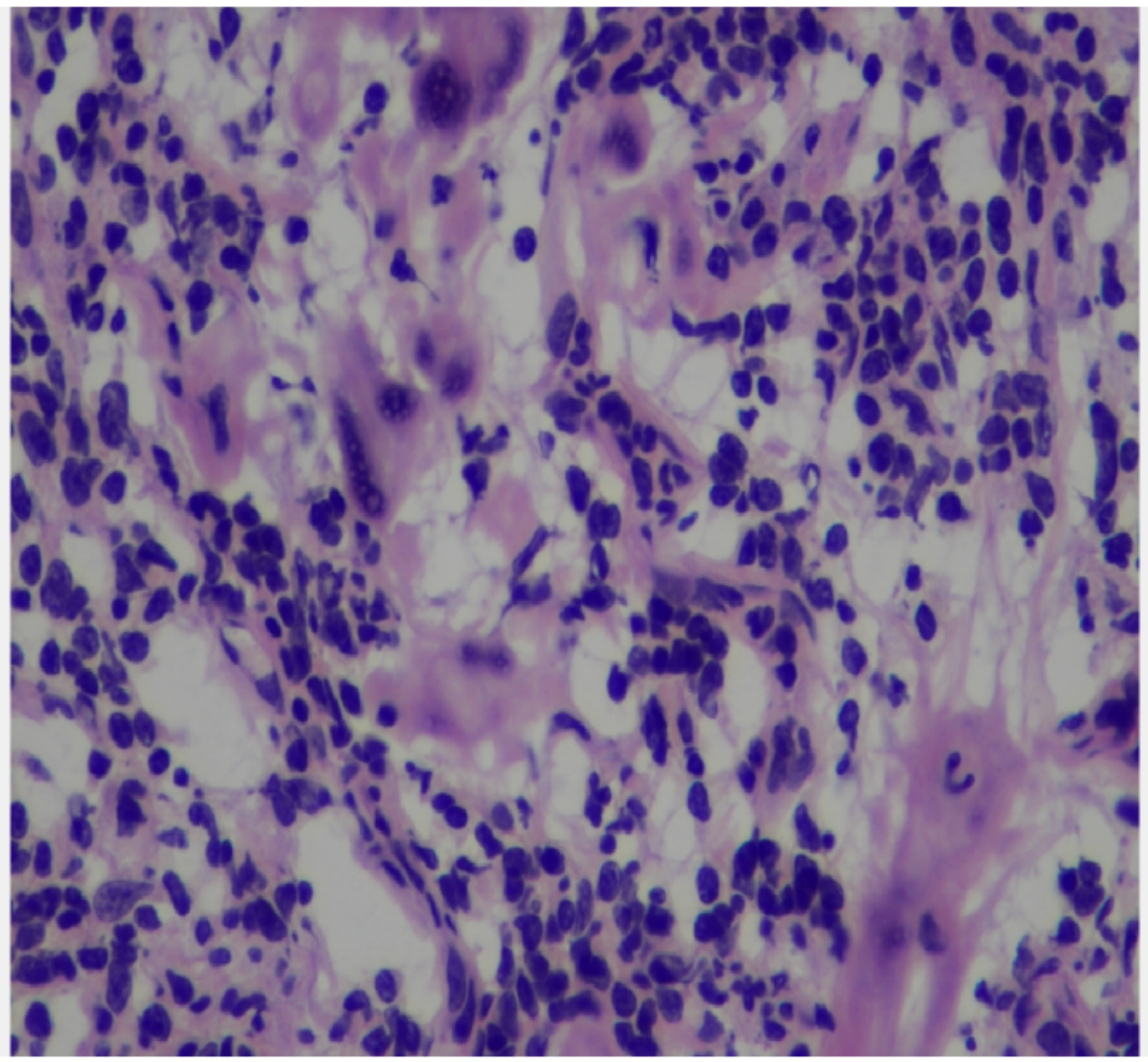

Figure 3

HE staining of inguinal lymph nodes. 


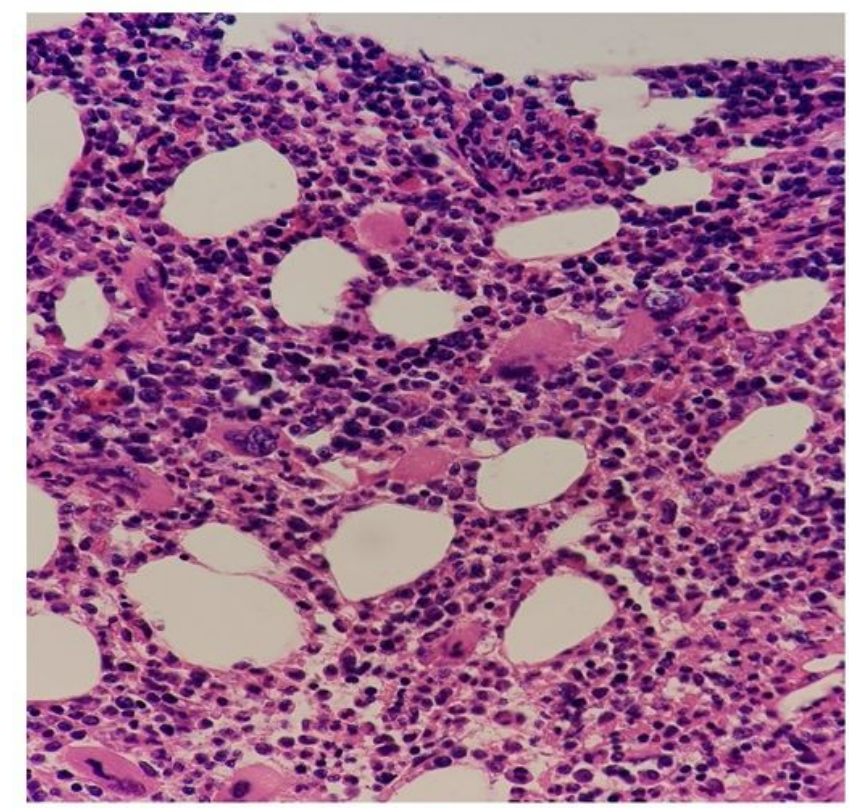

4.1

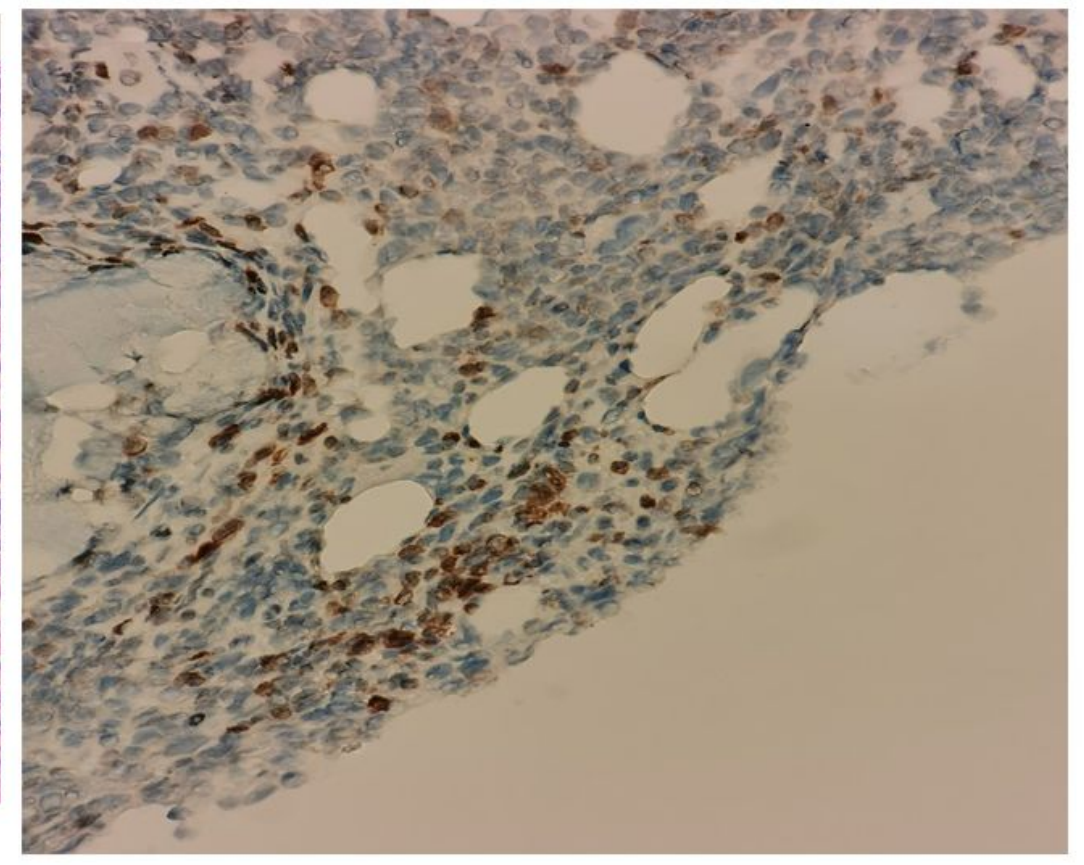

4.2

\section{Figure 4}

4-1: HE staining of bone marrow biopsy: The normal structure of the submitted lymph node was destroyed, and many small lymphocytes with a single shape were diffusely proliferated and infiltrated, and the cytoplasm was light and translucent, which were tumor cells. There were also large-sized immunoblasts scattered in the interstitium. High endothelial blood vessels Irregular hyperplasia with infiltration of eosinophils. 4-2: CD3 (positive), CD4 (negative), CD8 (positive, partly), CD20 (negative), CXCL-13 (negative), Bcl-6 (negative). 


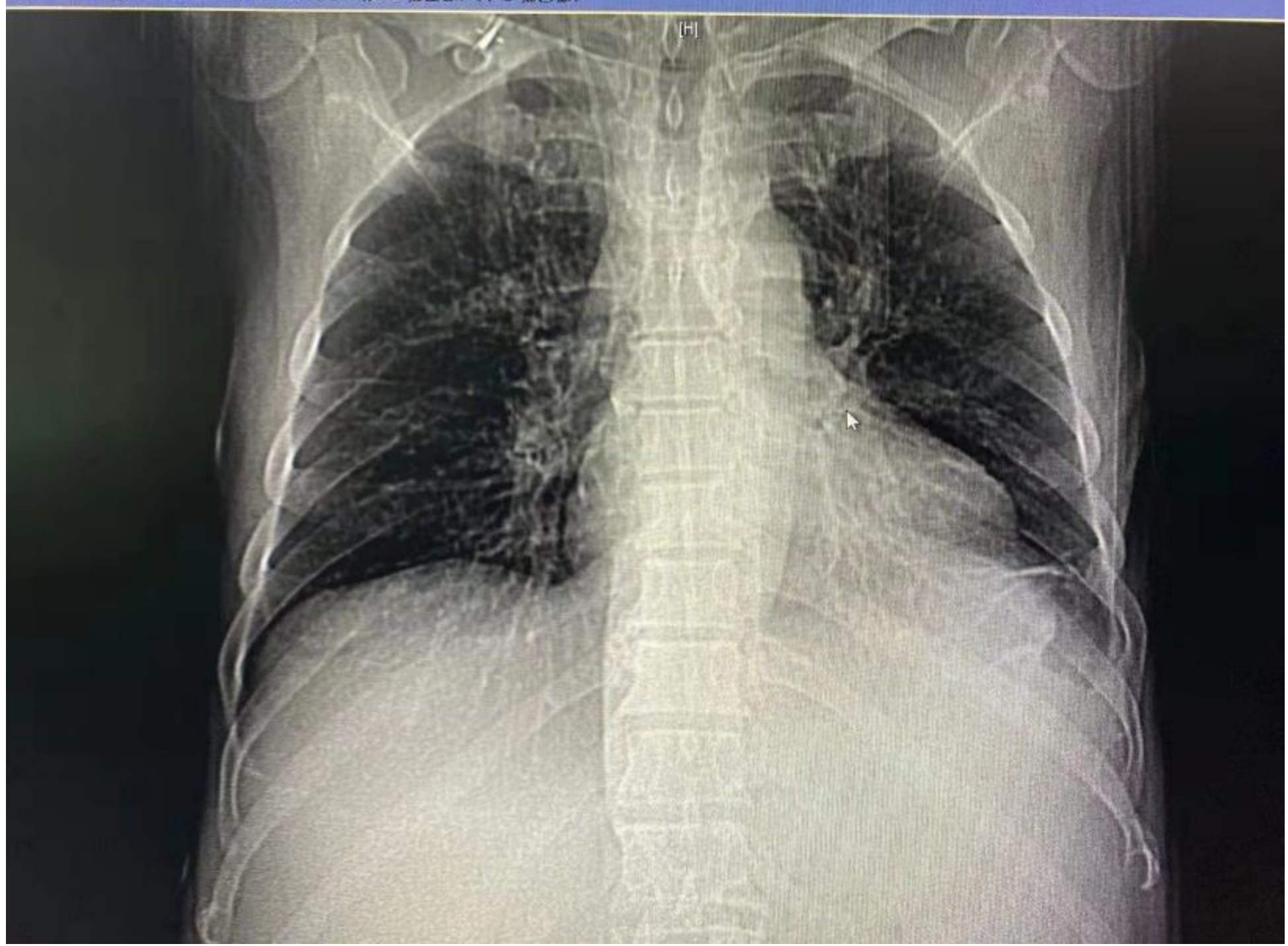

Figure 5

The patient's condition improved after active treatment. 